\title{
A Facile Method for Permanent and Functional Surface Modification of Poly(dimethylsiloxane)
}

\author{
Yuanzi Wu, ${ }^{\dagger}$ Yanyi Huang, ${ }^{\ddagger}$ and Hongwei Ma*, ${ }^{\dagger}$ \\ Department of Biomedical Engineering and Department of Advanced Materials and Nanotechnology, College of \\ Engineering, Peking University, Beijing 100871, P. R. China
}

Received February 27, 2007; E-mail: hongwei@coe.pku.edu.cn

Poly(dimethylsiloxane) (PDMS) is the choice of material for a wide range of applications, ${ }^{1-3}$ because PDMS has many advantageous properties such as chemical inertness, nontoxicity, ease of handling, and commercial availability. It is impossible, however, to have one material that meets all the individual needs of microfluidic systems, ${ }^{1}$ micro-electromechanical systems (MEMS), ${ }^{2}$ and cellular study. ${ }^{3}$ New materials have been developed to replace PDMS. For example, a photocurable perfluoropolyether (PFPE) was synthesized to fabricate microfludic devices that were organic solvent compatible. ${ }^{4,5}$ In a consensus, it is costly to develop a new elastomer for each individual need. And we believe surface modification of PDMS will be a cost-effective and time-saving strategy, if a facile method for surface modification can be developed, since surface modification retains the desired bulk properties of PDMS and reveals the need for new material development.

A number of strategies have been developed for PDMS surface modification, which can be divided into two categories, namely physisorption and chemical coupling. Physisorption of materials to PDMS surface, such as surfactants ${ }^{6}$ and polyelectrolytes ${ }^{7}$ are driven by hydrophobic force and electrostatic force, respectively. This simple method ensures PDMS based devices after modification perform well in situations that only require moderate density and thickness of coatings, and only to sustain low shear force.

Chemical coupling is stable but is difficult to achieve because PDMS is chemically inert, which is ironically one of its merits. Common for this approach, the first step is to apply high-energy bombardment (i.e., plasma) to PDMS surface, which results in a silicate layer with functional groups on the surface, such as $-\mathrm{OH}$ and $-\mathrm{NH}_{2}$. Those functional groups not only render the surface hydrophilicity but also allow further modification via chemical coupling. ${ }^{8}$ Chemical coupling has two problems: (1) Plasma treatment is easy but not sustainable; recovery of hydrophobicity of treated PDMS is well documented. ${ }^{9}$ High-energy bombardment also has the tendency to damage PDMS. Furthermore, this strategy is only applicable to planar structure because of its limited penetration depth. (2) Concentration gradient in "grafting to" strategy prevents the preparation of thick and dense films. ${ }^{10}$

We reported herein a facile method for permanent and functional surface modification of PDMS based on a commercial material. First, a vinyl-terminated initiator (v-initiator, part C in Scheme 1) was mixed with the viscous base and curing agent of Sylgard 184, resulting in an initiator integrated PDMS (iPDMS). The base is a poly(dimethyl-methylvinylsiloxane) prepolymer with small amount of platinum (Pt) catalyst (part A in Scheme 1) and the curing agent is a mixture of vinyl-endcapped PDMS precursors and poly(dimethyl-methylhydrogenosiloxane) precursors as cross-linkers (part B in Scheme 1). Upon mixing together (the so-called curing

$\dagger$ Department of Biomedical Engineering.

¥Department of Advanced Materials and Nanotechnology.
Scheme 1. Preparation of iPDMS and Permanent Surface Mondification of iPDMS via SI-ATRP

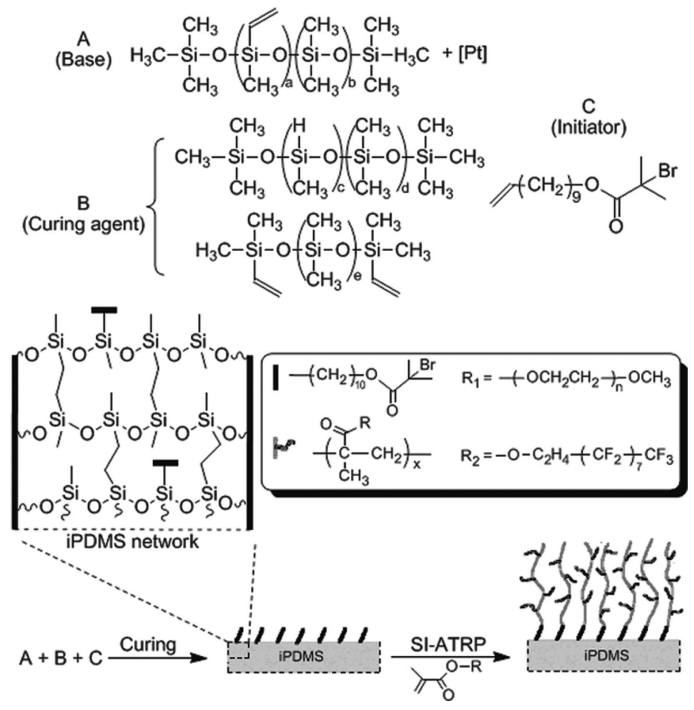

process), the vinyl groups and the hydrosilane hydrogens undergo a hydrosilylation reaction in the presence of Pt catalyst, which results in highly cross-linked three-dimensional networks.

It is a common practice to tune the mechanical property of PDMS by varying the ratio between $\mathrm{A}$ and $\mathrm{B} .{ }^{11}$ We reasoned that although the attachment of initiator to cross-linkers would evidently decrease the degree of cross-linking, one could introduce enough v-initiators into the network but only cause limited property change (i.e., mechanical property) by carefully choosing the ratio of $\mathrm{A} / \mathrm{B} / \mathrm{C}$. In fact, as a random cross-linking process, the network formation is not perfect even without component $\mathrm{C}$ and there is always a small amount $(<5 \%, w / w)^{12}$ of unreacted functional groups left.

We found that below a critical ratio of 10:1:0.5 (A/B/C) the mixture cured as regular PDMS (Young's modulus E $\sim 2.12 \mathrm{MPa}$, contact angle $\theta \sim 112^{\circ}$ ) and the resulting iPDMS (E $\sim 2.05 \mathrm{MPa}$, $\theta \sim 114^{\circ}$ ) was successfully used in replica molding. The key for successful surface modification was whether v-initiators would be presented at the surface. X-ray photoelectron spectroscopy (XPS) was applied to characterize the surface composition of iPDMS. Fresh iPDMS was extracted thoroughly with organic solvents to remove unreacted oligosiloxanes and trapped v-initiators. ${ }^{12}$ Survey scans of iPDMS showed a v-initiator unique $\mathrm{Br} 3 \mathrm{~d}$ peak at $71 \mathrm{eV}$ (Figure 1A). Three-dimensional XPS scans provided more information on the distribution of initiators in iPDMS: the $X-Y$ plane was characterized via sequential point-scan of a $3 \times 3$ square $(9$ points, step of $1 \mathrm{~mm}$ ). The calculated and experimental atomic concentrations (atom \%) agreed well for both PDMS and iPDMS for Si, C, and $\mathrm{O}$ (Table 1). The value of atom $\%$ for $\mathrm{Br}$ was lower than the 

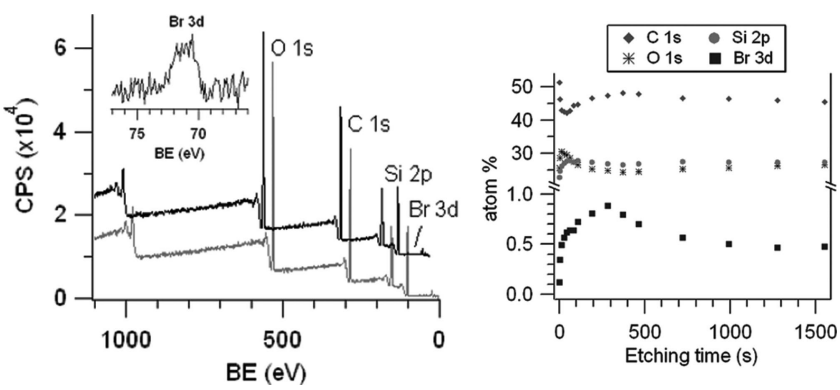

Figure 1. XPS confirmed the surface presentation of the vinyl-terminated initiator in iPDMS. (A) survey scans of PDMS (the gray curve) and iPDMS (the black curve, shifted in $X$-, $Y$-axes): CPS, counts per second; BE, binding energy. (B) XPS depth profile via etching for iPDMS.

Table 1. XPS Confirmed Uniform Surface Distribution of Initiators on iPDMS

\begin{tabular}{lllllll}
\hline & \multirow{2}{*}{$\begin{array}{c}\mathrm{A} / \mathrm{B} / \mathrm{C} \\
\text { (mass ratio) }\end{array}$} & & \multicolumn{4}{c}{ atom \% ${ }^{d}$} \\
\cline { 4 - 7 } & \multirow{2}{*}{$\mathrm{Si} / 1 / 0$} & calcd $^{a}$ & \multicolumn{1}{c}{$\mathrm{C}$} & \multicolumn{1}{c}{$\mathrm{O}$} & $\mathrm{Br}$ \\
\hline PDMS & 10.0 & 50.0 & 25.0 & \\
& & exptl $^{b}$ & $27.2 \pm 0.1$ & $49.4 \pm 0.1$ & $22.4 \pm 0.1$ & \\
iPDMS & $10 / 1 / 0.5$ & calcd $^{c}$ & 23.9 & 51.7 & 24.1 & 0.3 \\
& & exptl $^{b}$ & $26.7 \pm 0.1$ & $50.4 \pm 0.2$ & $22.8 \pm 0.1$ & 0.1 \\
\hline
\end{tabular}

${ }^{a}$ Atom $\%$ was calculated using $\left(\mathrm{SiOC}_{2}\right)$ as the repeat unit. ${ }^{b}$ Data averaged from 9 points, \pm standard error. ${ }^{c}$ The $M_{\mathrm{w}}$ for part A $+\mathrm{B}$ and part C were 74 and 305, respectively. ${ }^{d}$ Atom $\%$ was based on the survey scan of Si 2p (102 eV), C 1s (285 eV), O 1s (532 eV), and Br 3d (71 eV).

calculated value, which was attributed to the photobleaching effect of XPS on Br element and the loss of initiators during the curing process.

The Z-directional scans were accomplished via in situ etching of iPDMS surfaces (Figure 1B). The etching speed was $\sim 5 \mathrm{~nm} /$ min. Depth profiles of both PDMS and iPDMS concurrently showed the highest values of $\mathrm{Si}$ and $\mathrm{O}$, and the lowest values of $\mathrm{C}$ after 1 min etching. Interestingly, the highest atom $\%$ for $\mathrm{Br}$ appeared at $300 \mathrm{~s}$, which indicated that v-initiators were enriched at $\sim 25 \mathrm{~nm}$ below the surface. We could not exclude this enrichment was an artifact due to the vacuum process or Argon gun etching. Nevertheless, after this region of notable variations, the atom \% of all three major components reached a steady state. For PDMS, the atom \% for $\mathrm{O}, \mathrm{Si}$, and $\mathrm{C}$ were reasonably close to the calculated values (Table 1). For iPDMS, the atom $\%$ for $\mathrm{O}, \mathrm{Si}$, and $\mathrm{C}$ were $26 \%$, $27 \%$, and $45 \%$, respectively. The v-initiator unique element, $\mathrm{Br}$, had an atom $\%$ of $0.5 \%$. This small difference between the calculated and experimental values could be attributed to experimental error, or deviations of the final ratio of $\mathrm{A} / \mathrm{B} / \mathrm{C}$ in iPDMS network from the feed ratio of $\mathrm{A} / \mathrm{B} / \mathrm{C}$, especially when an extraction process was applied to remove unreacted precursors. Besides Sylgard 184 from Dow Corning, RTV 615 from GE and ELASTOSiL RT 601 from WACKER were also tested to be compatible with this iPDMS method.

Next, we carried out SI-ATRP of two representative monomers from iPDMS for permanent and functional surface coatings, namely oligo(ethylene glycol) methylmethacrylate(OEGMA) and $1 \mathrm{H}, 1 \mathrm{H}, 2 \mathrm{H}, 2 \mathrm{H}$ perfluorodecyl methacrylate (FMA). OEGMA was chosen for its demonstrated ability in improving the biocompatibility of PDMS, which was the key for the success of many bioMEMS devices. ${ }^{1,3}$ FMA was commonly used to create surfaces with ultralow surface energy. ${ }^{13}$ In Figure 2A, the characteristic F 1s peak at $690 \mathrm{eV}$ clearly indicated the success of polymerization and film deposition $(\sim 28$ $\mathrm{nm}$ after $1 \mathrm{~h} \mathrm{SIP,} \theta \approx 128^{\circ}$ ). The atom $\%$ for poly(FMA) coated iPDMS were (O) 7.1\%, (Si) 1.9\%, (C) 40.9\%, (F) 49.9\%, and (Br) $0.2 \%$, respectively, which were very close to theoretical values:
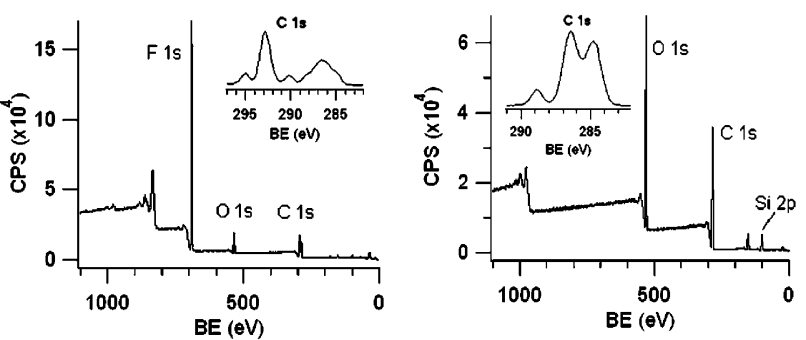

Figure 2. XPS confirmed successful surface modification from iPDMS via SI-ATRP: (A) survey scan of poly(FMA) coating, and (B) poly(OEGMA) coating. Inserted were the core scans of $F 1 \mathrm{~s}$ and $\mathrm{C} 1 \mathrm{~s}$.

(O) $6.7 \%$, (C) $36.7 \%$, and (F) $56.7 \%$. Surface grafting of OEGMA did not give such a distinct XPS signal but there was a significant atom $\%$ change due to the addition of poly(OEGMA) coating ( $\sim 50$ nm after 2 h SIP, $\theta \approx 48^{\circ}$ ): (O) $66.4 \%$, (Si) $5.1 \%$, and (C) $28.5 \%$ (Figure 2B), which were also close to theoretical values: (O) $66.7 \%$ and (C) $33.3 \%$.

Initiators of different structures have been immobilized onto PDMS surfaces by physisorption ${ }^{14}$ and chemical coupling. ${ }^{15}$ The iPDMS approach differentiates itself from the aforementioned approaches in that v-initiators are covalently integrated into the PDMS networks by a simple mixing procedure, which allows the permanent modification of intact microfluidic channels.

In conclusion, we demonstrated a simple yet effective method to realize permanent and functional surface modification of PDMS. The herein method relies on the creation of iPDMS and subsequent SI-ATRP from iPDMS, which renders PDMS tunable surface properties, for example, from very hydrophilic to very hydrophobic. This combination of iPDMS and SI-ATRP makes possible the application-directed surface modification of PDMS. And we believe this cost-effective method will improve the advancement of bioMEMS, microfluidics, and chips for cellular studies, where surface properties of PDMS plays an important role.

Acknowledgment. The authors thank Dr. Chunyang Xiong and Miss Chuanli Lin for Nanoindentation measurements. This work was supported by the NSFC grants (Grant 20604002) to H.M. and (Grant 20741002) to Y.H.

Supporting Information Available: Experiment details. This material is available free of charge via the Internet at http://pubs.acs.org.

\section{References}

(1) Whitesides, G. M. Nature 2006, 442, 368-373.

(2) Psaltis, D.; Quake, S. R.; Yang, C. H. Nature 2006, 442, 381-386.

(3) El-Ali, J.; Sorger, P. K.; Jensen, K. F. Nature 2006, 442, 403-411

(4) Rolland, J. P.; Van Dam, R. M.; Schorzman, D. A.; Quake, S. R.; DeSimone, J. M. J. Am. Chem. Soc. 2004, 126, 2322-2323.

(5) Huang, Y.; Castrataro, P.; Lee, C. C.; Quake, S. R. Lab Chip 2007, 7 , $24-26$.

(6) Huang, B.; Wu, H. K.; Bhaya, D.; Grossman, A.; Granier, S.; Kobilka B. K.; Zare, R. N. Science 2007, 315, 81-84.

(7) Liu, Y.; Fanguy, J. C.; Bledsoe, J. M.; Henry, C. S. Anal. Chem. 2000, 72, 5939-5944.

(8) Donzel, C.; Geissler, M.; Bernard, A.; Wolf, H.; Michel, B.; Hilborn, J.; Delamarche, E. Adv. Mater. 2001, 13, 1164

(9) Olah, A.; Hillborg, H.; Vancso, G. J. App. Surf. Sci. 2005, 239, 410423.

(10) Ma, H.; Wells, M.; Beebe, T. P., Jr.; Chilkoti, A. Adv. Funct. Mater 2005, 16, 640-648

(11) Schmid, H.; Michel, B. Macromolecules 2000, 33, 3042-3049.

(12) Lee, J. N.; Park, C.; Whitesides, G. M. Anal. Chem. 2003, 75, 65446554.

(13) Tsibouklis, J.; Nevell, T. G. Adv. Mater. 2003, 15, 647-650.

(14) Hu, S. W.; Brittain, W. J. Macromolecules 2005, 38, 6592-6597.

(15) Xiao, D. Q.; Van Le, T.; Wirth, M. J. Anal. Chem. 2004, 76, 2055-2061. JA071384X 


\title{
A Facile Method for Permanent and Functional Surface Modification of Poly(dimethylsiloxane)
}

\author{
Yuanzi $\mathrm{Wu}^{1}$, Yanyi Huang ${ }^{2}$, and Hongwei $\mathrm{Ma}^{1}$ * \\ ${ }^{1}$ Department of Biomedical Engineering, ${ }^{2}$ Department of Advanced Materials and \\ Nanotechnology, College of Engineering, Peking University, Beijing, 100871, P. R. \\ China
}

\section{Experimental:}

The vinyl terminated initiator (v-initiator, Undec-10-enyl 2-bromo-2methylpropanoate), the methyl terminated initiator (c-initiator, decyl 2-bromo-2methylpropanoate) and the thiol terminated initiator (s-initiator, $\omega$-mercaptoundecyl bromoisobutyrate) were obtained from HZDW (Hangzhou, China). Monomer oligo(ethylene glycol) methyl methacrylate (OEGMA) and 1H,1H,2H,2H-perfluorodecyl methacrylate (FMA) were purchased from Aldrich. Monomers were first passed through an aluminum oxide column to remove inhibitors.

PDMS preparation: Sylgard 184 from Dow Corning was used as a model elastomer. To prepare regular PDMS substrates, the viscous base and the curing agent (10:1 ratio by weight) were mixed well and cured at $80{ }^{\circ} \mathrm{C}$ for $2 \mathrm{~h}$.

To prepare v-initiator integrated PDMS (iPDMS), a third component, a v-initiator (part $\mathrm{C}$ in Scheme 1) was mixed well with the base and curing agent. It reacts with hydrosilane hydrogens in the presence of Pt catalyst, thus integrates in situ into the highly crosslinked three-dimensional networks. 
To prepare c-initiator and s-initiator integrated PDMS (cPDMS and sPDMS), cinitiator and s-initiator were used to replace the v-initiator as component $\mathrm{C}$.

SI-ATRP from iPDMS. All iPDMS disks were immersed in hexane for 24 hours to remove any physisorped and entrapped v-initiators. For SI-ATRP of OEGMA, the reaction mixture was prepared by mixing well of two parts. Part 1 was a transparent, pale-blue solution, prepared by adding a specified amount of $\mathrm{CuCl}_{2} / \mathrm{Bipy}(1: 2$ mole ratio) and a fixed amount monomer to $5 \mathrm{~mL}$ MilliQ-water. Part 2 was a colorless solution, prepared by adding a specified amount of ascorbic acid (AscA) to $5 \mathrm{~mL}$ MilliQ-water. After both solutions were deoxygenated, two parts were mixed together under nitrogen. The mixture was further deoxygenated and the resulting mixture was red in color due to the reduction of deactivator $\mathrm{Cu}(\mathrm{II}) / \mathrm{Bipy}$ complex to activator $\mathrm{Cu}(\mathrm{I}) / \mathrm{Bipy}$ complex. The resulting mixture had a mole ratio of monomer $/ \mathrm{CuCl}_{2} / \mathrm{Bipy} / \mathrm{AscA}=200 / 1 / 2 / 1$, with a feed $\left[\mathrm{CuCl}_{2}\right]$ of $2.76 \mathrm{mM}$. This mixture was then transferred to a reaction setup under nitrogen atmosphere and SIP was initiated and continued for $120 \mathrm{~min}$ at $\sim 25^{\circ} \mathrm{C}$. The polymerization was stopped when iPDMS was removed from the solution. Samples were thoroughly rinsed with methanol, MilliQ-water, and dried under flowing nitrogen before further treatment.

For SI-ATRP of FMA, $1.06 \mathrm{~g}$ FMA was added in $10 \mathrm{~mL}$ DMF by drops, and commixed by magnetic bar under deoxygenating protection for 20 minutes. Then $\mathrm{CuBr}$ (28mg), PMDETA(52mg), were added to the solution and mixed well resulting a homogeneous puce solution. The resulting mixture had a mole ratio of monomer/CuBr/PMDETA $=200 / 2 / 3$. After deoxygenated for 30 minutes, this mixture was then transferred to a reaction setup under nitrogen atmosphere. SIP was initiated and 
continued for 120 min at $\sim 25^{\circ} \mathrm{C}$. The polymerization was stopped when iPDMS was removed from the solution. Samples were thoroughly rinsed with methanol, MilliQ-water, and dried under flowing nitrogen before further treatment.

X-ray Photoelectron Spectroscopy. XPS (AXIS Ultra by Kratos Analytical, UK) was used to characterize the surface chemistry of PDMS before and after modification. Monochromatic $\mathrm{Al} \mathrm{K \alpha} \mathrm{X}$-rays $(1486.7 \mathrm{eV})$ were employed. The X-ray source was $2 \mathrm{~mm}$ nominal X-ray spot size operating at $15 \mathrm{kV}$ and12 mA for both survey and highresolution spectra. Neutralizer gun was on (1.85 A and 3.15 V). Survey spectra, from 0 to $1100 \mathrm{eV}$ binding energy (BE), were recorded at $160 \mathrm{eV}$ pass energy with an energy step of $1.0 \mathrm{eV}$, and a dwell time of $200 \mathrm{~ms}$. High-resolution spectra were recorded at $40 \mathrm{eV}$ pass energy with an energy step of $0.1 \mathrm{eV}$, and a dwell time of $500 \mathrm{~ms}$, with a typical average of 3 scans. The operating pressure of the spectrometer was typically $\sim 10^{-4} \mathrm{~Pa}$. For quantitative XPS measurements, both survey and high-resolution scans were first taken at an angle of $90^{\circ}$ defined as the angle between the collection axis of photoelectron analyzer and sample plane. All peaks were referenced to $\mathrm{C} 1 \mathrm{~s}\left(\mathrm{CH}_{\mathrm{x}}\right)$ at $285 \mathrm{eV}$ in the survey scan spectra and $\mathrm{C} 1 \mathrm{~s}\left(\underline{\mathrm{CH}_{\mathrm{x}}}\right)$ at $284.8 \mathrm{eV}$ in the deconvoluted high resolution $\mathrm{C} 1 \mathrm{~s}$ spectra. All data were collected and analyzed using software provided by the manufacturer.

The depth profile of PDMS and iPDMS were obtained by applying an Argon ion gun to etch the samples. The ion gun was operated at $4 \mathrm{kV}$ and $10 \mathrm{~mA}$ emission current, which gave an estimated etching speed of $\sim 5 \mathrm{~nm} / \mathrm{min}$. The operation pressure of the spectrometer was kept at $\sim 3.0 \times 10^{-3} \mathrm{~Pa}$. 
Ellipsometry. Film thickness was measured on a M-2000V spectroscopic ellipsometer (J. A. Woollam Co., Inc) at angles of $65^{\circ}, 70^{\circ}$ and $75^{\circ}$ and wavelengths from $400 \mathrm{~nm}$ to 800 $\mathrm{nm}$. Ellipsometric data were fitted for the thickness with material specific models, i.e., SAMs and poly(OEGMA) films with fixed (An, Bn) values of $(1.45,0.01)$ and $(1.46$, 0.01), respectively using a Cauchy layer model; FMA was fitted with a model provided by the manufacturer. The ellipsometric thickness for each sample was independently measured at three different locations and is reported as the average \pm standard error (SE).

Contact Angle. The sessile water contact angles $(\theta)$ were measured on an OCA2O goniometer (Dataphysics, US.) using deionized water. All substrates were rinsed with ethanol and dried under a stream of nitrogen before measurement of their contact angle. Three locations were randomly chosen for $\theta$ measurement and reported as average $\pm \mathrm{SE}$.

Nanoidentation Measurements. The Young's modulus (E) of PDMS and iPDMS were obtained from a TriboIndenter (Hysitron, US.).

\section{Results and Discussion.}

Hydrosilylation Chemistry. To prove that the v-initiator was incorporated into the threedimensional networks via the hydrosilylation chemistry, two control experiments were conducted: (1) c-initiator (Fig. S1A) was used to replace the v-initiator as component C and resulted in cPDMS when the A, B and $\mathrm{C}$ were mixed at a ratio of 10:1:0.5. XPS measurements of cPDMS before and after hexane extraction showed significant change of $\mathrm{Br}$ peak intensity (Fig. S1B and C): the At\% for $\mathrm{Br}$ were $0.1 \%$ and below XPS detection limit for cPDMS before and after hexane extraction, respectively. This was a strong evidence indicating that c-initiator was physically entrapped within the PDMS 
network due to the lack of a vinyl group for hydrosilylation reaction; (2) When the sinitiator was used to replace the $\mathrm{v}$-initiator as component $\mathrm{C}$, it was found that the $\mathrm{A}+\mathrm{B}+$ C mixture was unable to form a solid (i.e., fail to curing). We believe this is due to the fact that the Pt catalyst was poisoned by the thiol group presented in the s-initiator.

(A)

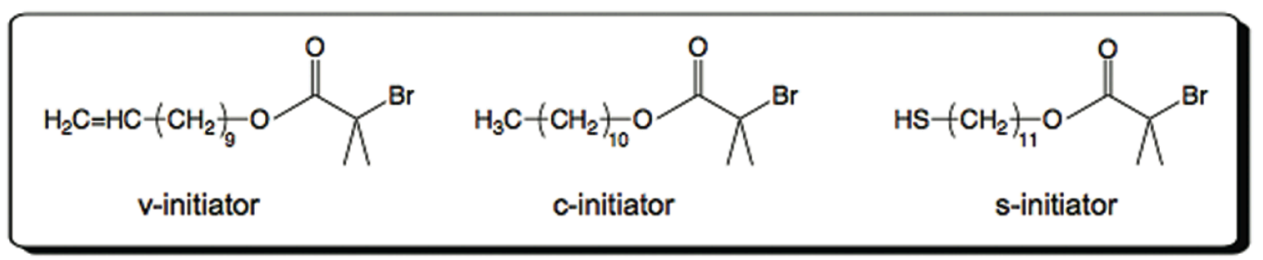

(B)

(C)
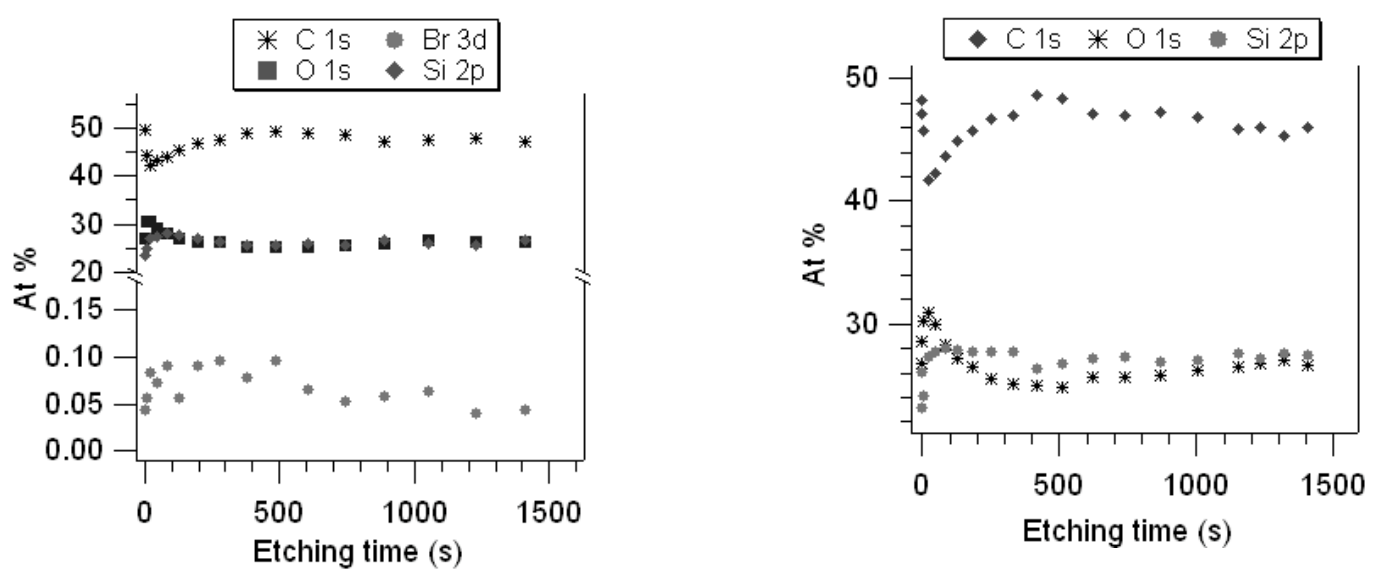

Figure S1. (A) chemical structures of three initiators. XPS depth profile of cPDMS before (B) and after (C) hexane extraction for $24 \mathrm{~h}$.

Thickness Estimation. It was difficutl to directly measure the resulting film thickness after SI-ATRP from iPDMS. Instead, an initiator modified silicon chip (Ma, H.; Li, D.; Sheng, X.; Zhao, B.; Chilkoti, A. Langmuir 2006, 22, 3751-3756) was polymerized together with iPDMS. And the film thickness on Si chip can be easily measured by ellipsometry, which was then used to approximate film thickness on iPDMS. 